\title{
Optimising pre-load study design for satiety assessment
}

\author{
M.R. Hobden ${ }^{1}$, L. Guérin-Deremaux ${ }^{2}$, I. Rowland ${ }^{1}$, G.R. Gibson ${ }^{1}$ and O.B. Kennedy ${ }^{1}$ \\ ${ }^{1}$ The University of Reading, Reading, Berkshire, RG6 6AP, UK and ${ }^{2}$ Roquette, Lestrem, France
}

Laboratory-based pre-load studies are often used to quantify the effects of foods and/or food ingredients on satiety and satiation. Important considerations when designing pre-load studies include, but are not limited to, the timings of the study meals in relation to habitual intake, the duration between the pre-load and ad libitum test meal, and the nutrient content and palatability of the study meals provided $^{(1)}$.

We conducted a pilot study to determine the satiety effects of, i) a gender-specific compared to a non gender-specific breakfast meal, and ii) the duration between pre-load and lunch (test meal), and the time of lunch provision. The palatability of the study meals and pre-load were also evaluated.

Forty healthy adult volunteers, with a BMI between 22.0 and $27.9 \mathrm{~kg} / \mathrm{m}^{2}$, attended a single study visit at the Hugh Sinclair Unit of Human Nutrition, University of Reading. Male volunteers $(n=20)$ received a $265.8 \mathrm{kcal}$ breakfast meal, equivalent to $\sim 10 \%$ of the population estimated average daily energy requirement for males ${ }^{(2)}$. Female volunteers received either the same $265 \cdot 8 \mathrm{kcal}$ breakfast $(n=10)$ or a lower energy, female-specific, breakfast of $213.9 \mathrm{kcal}(n=10)$, equivalent to $\sim 10 \%$ of the population estimated average daily energy requirement for females ${ }^{(2)}$. A $200 \mathrm{ml}$ orange juice pre-load was consumed $2 \mathrm{~h}$ after breakfast. Ten male volunteers followed protocol 1 (lunch provided at 12.00, $2 \mathrm{~h}$ after the pre-load) and thirty volunteers (male $n=10$, female $n=20$ ) followed protocol 2 (lunch provided at $13 \cdot 00,2 \cdot 5 \mathrm{~h}$ after the pre-load). Lunch was a pasta-based, single-course, meal provided ad libitum. Visual analogue scales (VAS) were completed at regular intervals throughout the study visit to assess subjective satiety and the palatability of the study meals and preload.

AUC satiety scores before lunch were $3.4 \pm 2.2 \mathrm{~mm} \times 15 \mathrm{~min}$ (mean $\pm \mathrm{SD}$ ) for protocol 1 and $4.5 \pm 1.7 \mathrm{~mm} \times 15 \mathrm{~min}$ for protocol 2 , however this was not found to be significantly different following analysis using one-way ANOVA. Pearson correlations revealed that the pre-load to lunch satiety scores of the male volunteers following protocol 2 were more strongly correlated with the satiety scores of the female volunteers following protocol 2 with gender-specific breakfast $(r=0 \cdot 51)$, compared to non gender-specific breakfast $(r=0.41)$. As expected, male volunteers consumed significantly more energy at lunch than the female volunteers $(P<0 \cdot 05)$. No significant differences were found between protocol 1 and protocol 2 for ad libitum energy intake at lunch. Additionally, no significant differences were found for energy intake at lunch between the female volunteers that consumed the gender-specific compared to non gender-specific breakfast. Mean scores for palatability, enjoyment, and taste of the breakfast, pre-load and lunch meals were all above 65 (out of 100) and did not differ between the study groups.

In conclusion, a gender-specific breakfast meal was found to improve the correlation between male and female volunteers for satiety measures assessed using VAS. Time of test meal provision (in relation to habitual meal times) and duration between pre-load and test meal have been evidenced to impact on satiety and energy intake ${ }^{(1)}$, however no significant effects were observed in the current study.

This research was financially supported by Roquette.

1. Blundell J, de Graaf C, Hulshof T, et al. (2010) Obesity reviews 11, 251-270.

2. Scientific Advisory Committee on Nutrition (2011) Dietary recommendations for energy. SACN republication; The Stationary Office: Norwich, UK. 